Katharina Jacob, Klaus-Peter Konerding \& Wolf-Andreas Liebert (Hg.). 2020. Sprache und Empathie. Beiträge zur Grundlegung eines linguistischen Forschungsprogramms (Sprache und Wissen 42). Berlin, Boston: De Gruyter. $631 \mathrm{~S}$.

Besprochen von Philipp Dreesen: Zürcher Hochschule für Angewandte Wissenschaften, Departement Angewandte Linguistik, Theaterstrasse 17, CH-8401 Winterthur, E-Mail: philipp.dreesen@zhaw.ch

https://doi.org/10.1515/zrs-2021-2075

Es ist leicht zu betonen, angesichts der Digitalisierung in Methodologie und Empirie in vielen Bereichen des Faches die Grundlagenforschung zu Sprache, Denken und Kommunikation nicht außer Acht zu lassen: Was sind die psychisch-kognitiven, sich im Sozialen äußernden Aspekte, die sprachliches Denken und Kommunikation ausmachen? Nicht leicht ist es, sich dieser Frage immer wieder erneut zu stellen und ein Desiderat herauszuarbeiten. Es ist das große Verdienst des Sammelbandes Sprache und Empathie. Beiträge zur Grundlegung eines linguistischen Forschungsprogramms, sich einem zentralen Baustein menschlicher Kommunikation zu nähern, der in der Linguistik bisher vernachlässigt wurde.

In den einleitenden „Allgemeine[n] Überlegungen zu einer Linguistik der Empathie“ weisen die Herausgeber`innen Katharina Jacob, Klaus-Peter Konerding und Wolf-Andreas Liebert darauf hin, dass der Begriff Empathie in vielen Disziplinen vorkommt, denn „Empathie ist ubiquitär“ (S. 5). Jedoch wird deutlich: Es handelt sich bei Sprache und Empathie nicht einfach um eine Form der frequenten Zwillingsformel Sprache und $X$. Vielmehr wird deutlich, wie sehr wir ein Konzept wie Mitgefühl, unterstelltes Common Ground, Hineinversetzen in andere, Situationsreflexion, Antizipierung, Explorationsfähigkeit und Ähnliches benötigen, um erklären zu können, wie Verständigung gelingt und gelingen kann. Letzteres zieht sich durch die Konzeption des Bandes, in dem neben der Grundlagenforschung auch die Relevanz für gesellschaftliche und anwendungsbezogene Bereiche von linguistischer Empathieexpertise hervorgehoben wird.

Der einleitende Text regt erfreulicherweise auch zu Widerspruch an, etwa wenn - das Konzept umkreisend - Empathie als „komplexe kommunikative Praktik“ (S. 2) bezeichnet wird oder vorsichtig behauptet wird, ,einige Dimensionen der tentativen Empathieimplementierung " fänden sich ansatzweise auch in „hoch entwickelte[n] Maschinen“ (S. 5). 


\section{Grundlegende Überlegungen}

Dem umfangreichen Band liegt eine überzeugende Dreiteilung mit zunächst grundlegenden Gedanken zum Thema, einer daran anschließenden Zusammenstellung von Fallstudien und schließlich potenzieller Forschungs- und Anwendungsfelder zugrunde. Im ersten Teil („Grundlegende Überlegungen zum Zusammenhang von Empathie, Kommunikation und Sprache“) werden die Grundlagen aus Philosophie, Ethnologie und Psychologie/Psychotherapie sowie zwei linguistische Zugänge kommunikationsbezogener Empathie dargelegt.

Thiemo Breyer differenziert in „Parameter und Reichweite der Empathie“ basierend auf seiner Monografie Verkörperte Intersubjektivität und Empathie (2015) zwischen leiblich-körperlichen, affektiv-emotionalen und kognitiven Dimensionen der Empathie. Dies erlaubt, auf Wechselwirkungen hinzuweisen, etwa bei zu Hass führendem leiblich-körperlichen Miterleben, welches wiederum das affektiv-leibliche Mitfühlen beeinflusst. Auf die Reichweite von Empathie geht Breyer in Bezug auf die Instrumentalisierung von Empathie ein (vgl. auch den Beitrag von Liebert in diesem Band).

An einer Stelle erwähnt Frank-M. Staemmler in seinem Beitrag „Selbsterleben, Bezogenheit und Resonanz" kurz die Diagnose der Empathielosigkeit (S. 36), wodurch das Selbstverständliche unmittelbar normativ wird. In der psychotherapeutischen Praxis wird Sprache zum Instrument nicht nur des Austausches, sondern auch der empathischen (möglichst reziproken) Beziehung zwischen Therapeut ${ }^{\star}$ in und Klientin.

Tномаs Stodulka unterzieht sein Fach, die Ethnografie, einer methodologischen Kritik, indem er dafür plädiert, etwa in der Feldforschung weder in „einen ,Codierungswahn““ (S. 66) zu verfallen, noch eine Verklärung von Empathie durch die Forschenden zu betreiben. Stodulka plädiert für eine Reflexion der Empathie in der Ethnografie durch methodische Fundierung und verweist auf die Forschungsleistung in anderen Fächern der vergangenen Jahre.

Ausgehend von der Fachgeschichte mit Schwerpunkt auf der Pragmatik fragt Klaus-Peter Konerding in seinem Beitrag nach den ,grundlegenden Voraussetzungen gelingender menschlicher Begegnung und Kooperation“ (S. 81). Pointiert wird aufgezeigt, dass Levinsons noch immer das Fach prägendem Neo-Griceismus ein Konzept wie Empathie schlicht fehle und sein Kommunikationsmodell somit - überspitzt formuliert - dem eines Psychopathen entspreche (S. 95). Unter Rückgriff auf die Psychologie/Psychiatrie und die Neurowissenschaften sei es möglich, die bisherige Modellierung von interaktionaler Kommunikation um den Faktor Empathie angemessen komplex zu ergänzen, so Konerding.

Wolf-Andreas Liebert setzt fachgeschichtlich an der Kulturwissenschaft und Hermeneutik an. Fritz Hermanns Arbeiten (z.B. 2007) zeigen die linguistische 
Qualität der Empathie als „Grundbegriff der Hermeneutik“, werden aber nun erst bei Liebert für die kulturwissenschaftlich orientierte Linguistik aus der ,Sackgasse der Spiegelneuronen' (vgl. S. 111) befreit und in ihrem explorativen Wert gestärkt. Untermauert wird dies mit Breithaupts (2017) Verbindung von Empathie und Narration, in Lieberts Beitrag primär bezogen auf biografische Hintergründe von Subjekten (Belebtes und Unbelebtes), mit denen wir empathisch sein können. Liebert gibt hiervon ausgehend einen vielversprechenden Ausblick auf die Idee eines „Empathiedispositivs“ zur Erfassung der selektiven Empathie: Wie werden wir sozialisiert in Empathiegebot und -verbot?

\section{Exemplarische Aspekte}

Der zweite Teil „Empathie in intrakultureller, interkultureller, multimodaler und Interspezies-Kommunikation" umfasst sehr unterschiedliche Aspekte, deren $\mathrm{Zu}$ sammenstellung sich vor allem aus der Differenz zu den anderen Teilen ergibt. Der erste Beitrag von MAXı KupETz basiert auf bereits publizierten empirischen Daten (z.B. 2015), die unter drei Dimensionen neu zusammengestellt werden: Situiertheit vom Empathiedarstellungen, Ressourcen von Empathiespielarten sowie Praktiken. Um Ressourcen geht es auch im Beitrag „Empathie und Interkulturalität“ von SABine RetTinger. Sie greift Breithaupts narratives Empathieverständnis auf und liest unter diesem Gesichtspunkt Richard Rortys (1989) Imagination von Solidarität neu. Dadurch wird der Blick auf Diskurse zu Migration und Interkulturalität geschärft, die ein letztlich anthropologisch begründetes Repertoire an fiktionalen und nicht-fiktionalen Textsorten umfassen, zum Zweck einer Horizonterweiterung durch Empathie und deren Irritationen. Wie dies misslingen kann, zeigt AndReas RothenhöFer in seinem Beitrag „Mehrfachadressierung, multimodale Empathiemanifestation und Blockade“ am Beispiel der Talkshow Hart aber fair von 2014 zur Frage „Deutschland und der Islam - wie passt das zusammen?“. Medienspezifische Vorbedingungen wie Sendungstitel, Schnitt, Moderation, Auswahl an Talkshowgästen und weitere dramaturgische Faktoren verunmöglichen nahezu die Ausbildung von Empathie. Es entsteht ein Teufelskreis (S. 244), indem die muslimischen Talkshowgäste durch die Dramaturgie des Sendungsverlaufs nonverbal Resignation und Kooperationsunwilligkeit vermitteln und damit die Chance zur Einfühlung seitens der Mehrheitsgesellschaft verringert wird.

Die selektive Empathie wird von Pamela Steen in Bezug auf das Mensch-TierVerhalten aufgegriffen. Exemplarisch werden Erzählungen von Jägerinnen untersucht, in denen vor allem der Tötungs- und Sterbensprozess empathisch reflektiert wird; kontrastiert wird dies durch die punktuelle Empathieevozierung durch 
Tierleid in einem Video der Tierschutzorganisation PETA. Um „Empathisieren“ mit Pferden geht es im Artikel von Heike Rettig, die sich mit kommunikativen Praktiken von Reittrainerinnen befasst. Im Ergebnis lassen sich u.a. Zuschreibungen von inneren Zuständen des Pferdes oder wechselseitige Gefühlsübertragungen zwischen Reiterin und Reittier feststellen.

\section{Relevanz}

Der dritte Teil trägt den Titel „Empathie und ihre Relevanz in exemplarischen gesellschaftlichen Kommunikationsbereichen" und zielt auf Konkretion und Anwendungsperspektiven. Hier sind vor allem erste Ideen, Forschungsskizzen und Fingerübungen versammelt.

Ein Autor^innenkollektiv (Ekkehard Felder, Andreas Deutsch, Friedemann Vogel, Stefan Höfler, Christina Hörth, Aza Gleichmann, Janine Luth, Ina Pick, Hans Kudlich \& Lars Bülow) befasst sich mit „Empathie im Recht“. Den von einzelnen Autor`innen verantworteten Teilkapiteln und dort ausgemachten linguistischen Desiderata ist wegen ihrer Kürze teilweise nur schwer zu folgen, teilweise fehlt hier der Platz, um ihnen gerecht zu werden. Wichtig erscheint bei der starken Betonung von Empathie gerade auch, deren Grenze und Verhältnismäßigkeit zu sehen, etwa in der juristischen Ausbildung (S. 364-367).

PAvla SchäfER stellt ausführlich das Verhältnis von Vertrauen und Empathie dar und kommt zum Ergebnis mehrfach reziproker Wechselwirkungen. Die exemplarischen Befunde einer korpusbasierten Analyse von Online-Bewertungen zur Arzt-Patienten-Kommunikation geschieht vor dem Hintergrund eines neues Masterplans Medizinstudium 2020, in dem soziale und kommunikative Kompetenzen mehr Gewicht bekommen sollen.

Michael Bender \& Nina Janich plädieren in einer Forschungsskizze für „Leitlinien für ein mögliches zukünftiges Forschungsprogramm Empathie in der Wissenschaftskommunikation“ (S. 422). Dabei weisen sie, illustriert durch ausgewählte Interaktionen zwischen Expert*innen und Lai`innen, nicht zuletzt darauf hin, dass es eine kaum zurückzuweisende Empathieerwartung an die Wissenschaftskommunikation gibt.

Josef KLEIN betrachtet in „Empathie als politische Ressource“ das verbale Werben um die Empathie für Dritte in politischen Kontexten. Die zusammengestellten Beispiele sind sprechend, die Analysen ähneln eher Paraphrasen.

Helmuth Ebert \& Edith Münch versuchen sich in ihrem Beitrag „Empathie in der Unternehmens- und Veränderungskommunikation“ an einer Meta-Analyse zu Empathiebezügen in Handbüchern zur Organisationskommunikation. 
Dass Empathie in der schulischen Bildung eine auszubildende Kompetenz sein könnte, befinden Jörg Kilian \& Konstanze Marx. Vor dem Hintergrund des bereits hohen Status von Empathie in der Lehramtsausbildung werden als Anregung Empathievermittlungsübungen für den Unterricht vorgestellt, die der häufig computervermittelten Kommunikation in der Lebenswelt von Schüler`innen gerecht werden soll.

Der Beitrag „Sprachkritik und Empathie“ von Kristin Kuck \& Kersten Sven Rотн gibt einen Einblick, wie die Einsendungen zur sprachkritischen Aktion Unwort des Jahres verfasst sind. Belege aus dem Korpus von ca. 30.000 Texten (1991 bis 2011) zeigen, analytisch aufbereitet, wie die Absender*innen zwischen sich und den Adressat*innen Empathiebezüge herstellen und dadurch ihr Anliegen argumentativ-empathisch transportieren.

Literarische Texte unterstützen u.a. durch Perspektivübernahme die Ausbildung von neuen Erfahrungen, davon gehen Berbeli Wanning \& Anna Mattfeldt in ihrem Beitrag zur Rolle der Empathie beim Verstehen fiktionaler Texte aus. Die Autorinnen diskutieren, wie die Perspektivübernahme von sprechenden Tieren in Kinder-, Jugend- und Erwachsenenliteratur über die fiktionale Welt hinaus in der Realität zur Empathieentwicklung genutzt werden kann.

Der abschließende Beitrag stammt von Paul Reszke. Er untersucht zwei Reden und den offiziellen Katalog zur 14. documenta (Kassel, Athen) mit dem Ziel, im textuellen Nahebringen von Kunstwerken eine „kunstkommunikationsspezifische[n] Empathie“ (S. 599f.) aufzuzeigen, um Empathie als zentrales Konzept der Wissensdomäne Kunst nachzuweisen.

\section{Fazit}

Der Sammelband rückt Empathie ins interdisziplinäre Licht der Linguistik. Bei aller Empathie und forschungsstrategischem Verständnis für die Besetzung des Feldes ist doch kritisch anzumerken, dass der Sammelband in seinem Titel und in einigen Beiträgen der Grundlegung eines linguistischen Forschungsprogramms weit vorausgreift. Was zur Grundsteinlegung noch fehlt, sind z.B. die Expertise in ontogenetischer Entwicklung sowie die theologische und historische Einordnung des Gegenstands. Hinsichtlich der selektiven Empathie wären wohl die Diskussionen der Feministischen Theorie zu berücksichtigen (etwa in Tong \& Botts 2018). Schließlich kann angemerkt werden, dass bei aller Skepsis gegenüber einer moralphilosophischen Verengung des Empathiekonzepts nicht unreflektiert bleiben sollte, inwiefern unser (selektives) Mitgefühl als Forschende gegenüber z.B. Unterdrückten ein wesentliches Kriterium bei der Entdeckung unserer Forschungsobjekte ist (Walzer 2000; Dreesen 2019). 
Nun, zu sagen, was alles noch fehlt, ist leicht. Noch leichter ist es, zu konstatieren: Der Sammelband zeigt eindrücklich, dass Forschungsprogramme zu Sprache und Empathie hochrelevant und hochwillkommen sind.

\section{Literatur}

Breithaupt, Fritz. 2009. Kulturen der Empathie. Frankfurt/Main: Suhrkamp.

Breyer, Thiemo. 2015. Verkörperte Intersubjektivität und Empathie. Philosophisch-anthropologische Untersuchungen. Frankfurt/Main: Klostermann.

Dreesen, Philipp. 2019. Politische Sprachkritik und das gute Auge. Zum Verhältnis von Wahrnehmen, Gesellschaftstheorie und Kritik. In: Aptum. Zeitschrift für Sprachkritik und Sprachkultur 15(2), 173-183.

Hermanns, Fritz. 2007. Empathie. Zu einem Grundbegriff der Hermeneutik. In: Fritz Hermanns \& Werner Holly (Hg.): Linguistische Hermeneutik. Theorie und Praxis des Verstehens und Interpretierens (Germanistische Linguistik 272). Tübingen: Niemeyer, 127-172.

Kupetz, Maxi. 2015. Empathie im Gespräch - Eine interaktionslinguistische Perspektive. Tübingen: Stauffenburg.

Rorty, Richard. 1989. Contingency, Irony, and Solidarity. Cambridge: Cambridge University Press. Tong, Rosemarie \& Tina Fernandes. 2018. Feminist Thought: A More Comprehensive Introduction. 5. Aufl. Boulder, CO: Routledge.

Walzer, Michael. 2000. Mut, Mitleid und ein gutes Auge. Tugenden der Sozialkritik und der Nutzen von Gesellschaftstheorie. In: Deutsche Zeitschrift für Philosophie 48(5), 709-718. 